\title{
A Heuristic Moving Vehicle Location Prediction Technique Via Optimal Paths Selection with Aid of Genetic Algorithm and Feed Forward Back Propagation Neural Network
}

\author{
${ }^{1}$ Baby Anitha, E. and ${ }^{2}$ K. Duraiswamy \\ ${ }^{1}$ Department of CSE, K.S.R. College of Engineering, \\ Tiruchengode, Namakkal District, Tamilnadu, India \\ ${ }^{2}$ Department of CSE, K.S. Rangasamy College of Technology, \\ Tiruchengode, Namakkal District, Tamilnadu, India
}

Received 2012-07-13, Revised 2012-10-23; Accepted 2012-12-12

\begin{abstract}
The moving object or vehicle location prediction based on their spatial and temporal information is an important task in many applications. Different methods were utilized for performing the vehicle movement detection and prediction process. In such works, there is a lack of analysis in predicting the vehicles location in current as well as in future. Moreover, such methods compute the vehicles movement by finding the topological relationships among trajectories and locations, whereas the representative GPS points are determined by the $30 \mathrm{~m}$ circular window. Due to this process, the performance of the method is degraded because such $30 \mathrm{~m}$ circular window is selected by calculating the error range in the given input image and such error range may vary from image to image. To reduce the drawback presented in the existing method, in this study a heuristic moving vehicle location prediction algorithm is proposed. The proposed heuristic algorithm mainly comprises two techniques namely, optimization GA algorithm and FFBNN. In this proposed technique, initially the vehicles frequent paths are collected by monitoring all the vehicles movement in a specific period. Among the frequent paths, the vehicles optimal paths are computed by the GA algorithm. The selected optimal paths for each vehicle are utilized to train the FFBNN. The well trained FFBNN is then utilized to find the vehicle movement from the current location. By combining the proposed heuristic algorithm with GA and FFBNN, the vehicles location is predicted efficiently. The implementation result shows the effectiveness of the proposed heuristic algorithm in predicting the vehicles future location from the current location. The performance of the heuristic algorithm is evaluated by comparing the result with the RBF classifier. The comparison result shows our proposed technique acquires an accurate vehicle location prediction ratio than the RBF prediction ratio, in terms of accuracy.
\end{abstract}

Keywords: Moving Vehicle Location Prediction, Genetic Algorithm (GA), Feed Forward Back Propagation Neural Network (FFBNN), Frequent Paths, Radial Basis Function (RBF)

\section{INTRODUCTION}

In the recent years, it is found that data mining and knowledge discovery has become some of the popular fields of researches. An important subset of this research is looking at the particular semantics of space and time and the approach in which they can be sensibly accommodated into data mining algorithms (Roddick et al., 2004). Spatial data mining comes into picture when the real data establishes a relation with spatial data. In other words, spatial data mining is the application of data mining processes to spatial data (Li and Wang, 2005). Spatial data mining is the technique of extracting implicit knowledge, spatial relations, or other patterns that are not Tamilnadu, India 
explicitly stored in spatial databases (Buang et al., 2011). Spatial data mining has deep roots in both traditional spatial analysis fields (such as spatial statistics, analytical cartography, exploratory data analysis) and various data mining fields in statistics and computer science (such as clustering, classification, association rule mining, information visualization and visual analytics) (Mennis and Guo, 2009).

The objects that are characterized by spatial location and/or expansion as well as by numerous non-spatial attributes are contained in the spatial database (Ester et al., 2001). In spatial data mining, the prediction of moving objects location based on the time series is a significant task in several applications such as wireless based, location-aware devices and networks of sensors and traffic analysis. The need for analysis, modeling and processing of traffic data is of much concern as the amount of moving vehicles are increasing speedily every day. Likewise, the extraction of additional information about traffic conditions, optional routes and possible prediction of troublesome situations, such as traffic jams, becomes necessary (Brakatsoulas et al., 2004). Mathematical study of spatial objects and its geometric properties which are preserved under deformations such as twisting and stretching are used in investigating the topology of a spatial object. The study of the topological changes of spatial objects over time, i.e., of time dependent geometries called moving objects, is important in several applications such as Geographical Information Systems (GIS), spatiotemporal databases, the processing of animation images in multimedia applications and the topology control of Wireless Sensor Networks (WSN) (Liu and Schneider, 2010).

With embedded GPS devices and other sensors the tracking of moving objects, whether it is a tiny cell phone or a giant ocean liner, is becoming increasingly accessible. Such enormous amount of data on moving objects poses immense challenges on effective and scalable analysis of such data and exploration of its applications (Li et al., 2006). The tracking data is obtained by sampling the positions using typically GPS to produce data that in database terms is commonly referred to as trajectories (Brakatsoulas et al., 2005). Recent progress on satellite, sensor, RFID, video and wireless technologies has made it possible to systematically track object movements and collect huge amounts of trajectory data, e.g., animal movement data, vessel positioning data and hurricane tracking data (Lee et al., 2008). It is important to know the approximate position of a mobile user in order to operate it, for this the knowledge about the positions of mobile objects has led to the location-based services and applications (Monreale et al., 2009).
In Mobile and Spatiotemporal databases, monitoring of continuous spatial queries over moving objects is necessary in several applications such as Public Transportation, Logistics, Location Based Services and more. In many applications, the moving vehicles location prediction plays an important role. Many methods were developed to find the vehicle location, which are briefly discussed in the following section. In such works, there is a lack of analysis in predicting the vehicles location. The vehicles location is predicted by finding the topological relationships among trajectories and locations and also the GPS points are determined by 30 $\mathrm{m}$ circular window from the image error range. The error range of the image is not static; it varies from image to image. So this technique is not applicable for all images. Moreover, there is no standardization available for selecting the suitable input image. If all the aforesaid drawbacks in the literary works are solved, then the moving vehicles location prediction performance is improved with higher accuracy.

In this study, a heuristic moving vehicle location prediction algorithm is proposed to predict the vehicles future location from the current location. The proposed technique is based on Genetic Algorithm (GA) and Feed Forward Back propagation Neural Network (FFBNN). The vehicles optimal paths are determined by one of the most popular optimization techniques called Genetic Algorithm (GA). The vehicles optimal paths are then used to train the FFBNN and this well trained FFBNN are utilized to predict the vehicles future location by knowing the vehicles current location. The rest of the study is organized as follows: Section 2 reviews the related works with respect to the proposed method. Section 3 discusses the proposed heuristic algorithm, which is detailed in its subsections. Section 4 discusses about the implementation results and section 5 concludes the study.

\subsection{Related Works}

A handful of research works available in the literature deals about the moving objects or moving vehicles location prediction techniques.

Mennis and Guo (2009) have proposed a graph-based approach that treats trajectory data as a complex network. They have also developed a technique that establishes the topological associations among trajectories and locations and utilizes a spatially constrained graph partitioning method to discover natural regions defined by trajectories which are within the context of vehicle movements. Moreover, it is been found that the discovered hierarchical regions can effectively facilitate the understanding of trajectory patterns and discovered the trajectory clusters that the existing methods failed to find. 
Pena and Santos (2011) have proposed an approach to store data about moving objects and also discussed regarding the key concepts associated in moving objects and their characteristics. For the analysis of moving objects, an overview of the existing data mining techniques and some future guidelines has also been presented.

Liu and Schneider (2011) have developed a model which determines the topological changes of a complex moving region through snapshots called observations. They have introduced a two phase strategy: the first phase partitions the observations into several evaluation units and uniquely maps a unit before the change to exactly one unit after the modification and the second phase interprets the topological change by integrating all basic topological changes from evaluation units.

Shaw and Gopalan (2011) have derived an application of the modified priori algorithm in coordinate sets of trajectories to find the frequent tranjectory coordinates. In order to reduce the unnecessary search time and space, certain additional steps have been added in the algorithm to prune the coordinate sets generated. They have also explained the basics of data origination, database structure to hold the coordinate datasets and the implementation of the algorithm with the object oriented programming language by an illustration. Their technique can be applied to interesting game domains to find the recurrent trajectory of an object shot by a player which follows a trajectory path.

Satpathy et al. (2011) have delivered a concise analysis of the trajectory data mining. There are still open research issues which are still unexplored. Hence, in their study, certain future works and research challenges have been reported.

\section{MATERIALS AND METHODS}

\subsection{Proposed Heuristic Moving Vehicle Location Prediction Technique}

Here, a method for predicting the vehicles future location is proposed. The proposed heuristic prediction technique consists of four stages namely, frequent paths selection, optimal paths prediction for each vehicle by GA, optimal paths training in FFBNN and vehicle future location prediction by FFBNN. This proposed method predicts the vehicles location by finding the vehicles frequent paths and allocating weights to each of the nodes (junctions). Each path's frequent values and node's weight values are utilized to find the vehicles optimal paths via the optimization algorithm GA. The optimal paths from the GA are then utilized in the FFBNN training and testing process. The structure of proposed heuristic technique is illustrated in Fig. 1.
In Fig. 1, initially the vehicles visiting paths in different time periods are collected and then we compute frequent paths for each vehicle. After that, the frequent paths are given to the optimization process to select most frequent paths for each vehicle. The most frequent paths from the optimization process is given to the FFBNN for performing training whereas this well trained FFBNN is utilized in the vehicles future location prediction.

\subsection{Frequent Paths Selection}

In this proposed method, the graph $G$ is designed with $n$ number of nodes (junctions) and the nodes are connected by the paths. The number of vehicles in the designed graph is represented as $\mathrm{V}=\left\{\mathrm{v}_{1}, \mathrm{v}_{2}, \ldots, \mathrm{v}_{\mathrm{m}}\right\}$, where, $\mathrm{m}$ is a value based on the number of vehicles that we are considered and the paths are denoted as $\mathrm{P}=\left\{\mathrm{p}_{1}\right.$, $\left.\mathrm{p}_{2}, \ldots \mathrm{p}_{\mathrm{z}}\right\}$. Moreover, the weights $\mathrm{W}_{\mathrm{n}}$ for nodes are assigned within the arbitrary integer values $[1, w]$. In a different time period $\mathrm{T}=\left\{\mathrm{t}_{1}, \mathrm{t}_{2}, \ldots \mathrm{t}_{\mathrm{i}}\right\}$, the vehicles $\mathrm{v}_{\mathrm{m}}$ visited paths need to be collected. The designed graph is illustrated in Fig. 2.

The vehicle $v_{1}$ initially start traversing at the node 1 in a time period $t_{1}$ and its next visit to the node 2 by utilizing the path $1->2$ and subsequently it visit the nodes 3 and 4 via the paths 2->3 and 3->4 respectively.

In this same time period $t_{1}$, the other vehicle's traversing paths are also to be computed. The similar process is repeated for all vehicles in different time periods and the collected paths from the aforementioned graph in three time periods are listed in the Table $\mathbf{1}$.

As can be seen from the Table 1, the vehicles are utilizing different paths to reach the same target node in different time periods. Similarly in different time periods, the vehicles $\mathrm{v}_{\mathrm{m}}$ visited paths are collected and the frequency value of path visited more than once by the particular vehicle $v_{m}$ is calculated. Based on the frequency value, the vehicle $v_{m}$ frequently visited paths $p$ is computed and an index value is allocated for each computed frequent path. The number of frequent paths for the vehicle $v_{m}$ is denoted as $\mathrm{f}_{\mathrm{P}}^{\mathrm{vm}}=\left\{\mathrm{p}_{1}, \mathrm{p}_{2}, \ldots \mathrm{p}_{\mathrm{j}}\right\}$ and the corresponding index value for the frequent paths is represented as $I^{\mathrm{vm}}=\left\{i_{1}, i_{2}, \ldots i_{x}\right\}$, where is a value based on the number of selected frequent paths for the vehicle $\mathrm{v}_{\mathrm{m}}$.

\subsection{Optimal Path Prediction by GA}

To find the precise future location of the vehicles, we select the optimal paths from the number of frequent paths $f_{p}$. The optimal paths selection by GA finds the precise paths for all vehicles utilized to identify the vehicles future location. Genetic algorithm offers better solution to a problem by considering the best combination of input variables. 


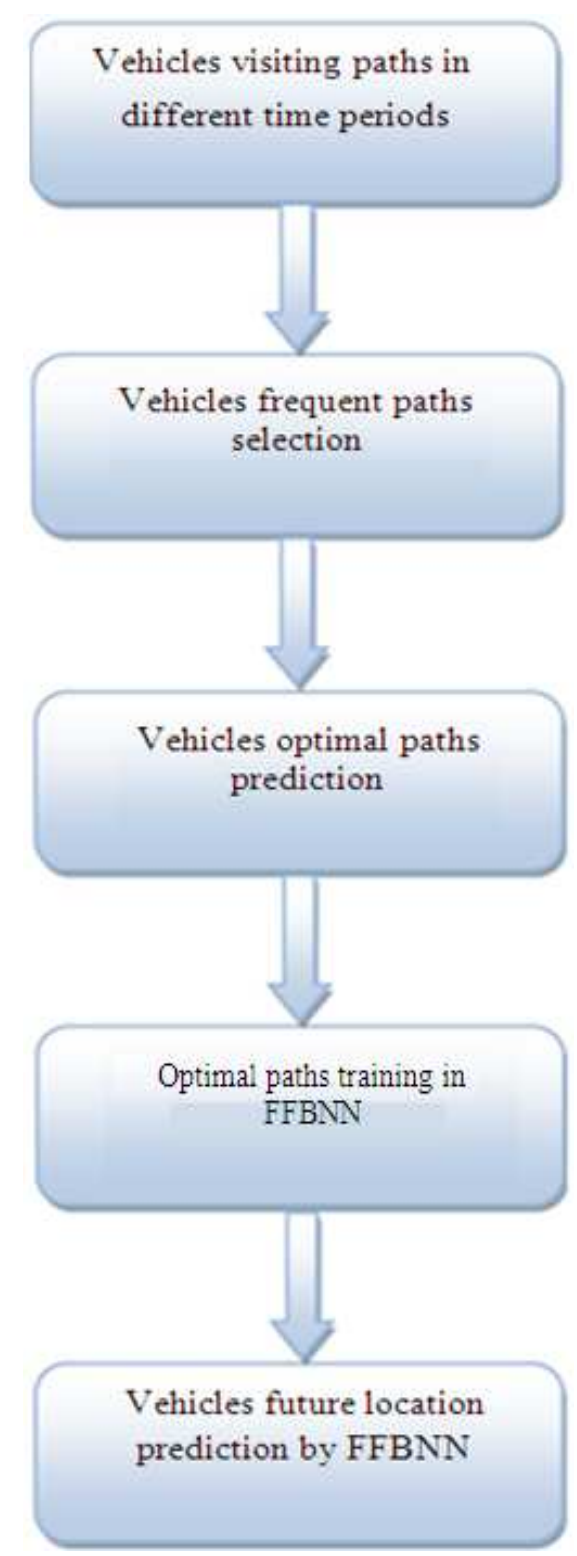

Fig. 1. Structure of proposed heuristic moving vehicle location prediction technique

It begins with the definition of the problem to solve and creates an objective function to compute the possible candidate solutions (chromosomes), i.e., the objective function is the way of identifying the individual producing the best result. To acquire the best solution, the threshold selection by GA carries out five basic processes: (i) Chromosome Generation, (ii) Computing fitness function, (iii) Crossover, (iv) Mutation and (v) Termination.

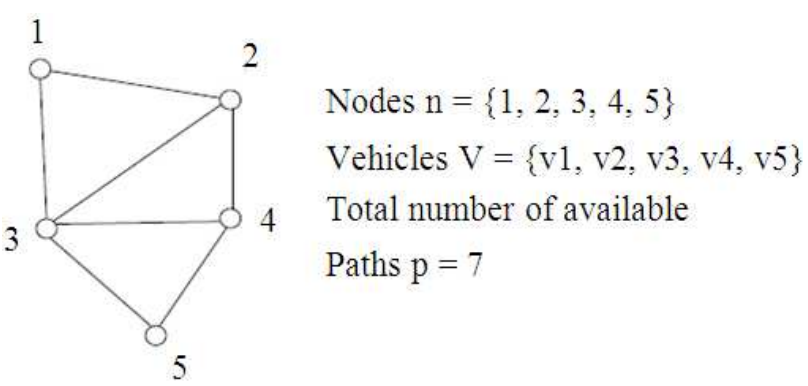

Fig. 2. Sample designed graph

\subsection{Generation of Chromosomes}

Here, an initial population of $\mathrm{N}$ individuals i.e., chromosomes are generated arbitrarily. The chromosomes are represented by binary string, where each binary position of the chromosome denotes a gene. Here, we generate the chromosomes as follows Eq. 1:

$\mathrm{c}^{\mathrm{v}_{\mathrm{m}} 1}=\left\{\mathrm{i}_{0}, \mathrm{i}_{1}, \mathrm{i}_{2}, \cdots \mathrm{i}_{\mathrm{x}}\right\}^{\mathrm{v}_{\mathrm{m}}}$
$0 \leq 1 \leq \mathrm{n}_{\mathrm{c}}-1$

In Eq. $1, \mathrm{v}^{\mathrm{cm}}$ is the $\mathrm{I}^{\text {th }}$ chromosome of the vehicle $\mathrm{v}_{\mathrm{m}}$ and $\mathrm{n}_{\mathrm{c}}$ is the number of chromosomes that are generated. Also, each gene value in the chromosome $i_{x}$ $\in \mathrm{c}^{\mathrm{vmI}}$ is an index value of the vehicle $\mathrm{v}_{\mathrm{m}}$ frequent paths. Among the number of generated chromosomes, the best chromosomes are selected based on their fitness value. This fitness function calculation for the $\mathrm{I}^{\text {th }}$ chromosome is described below.

\subsection{Fitness Function}

In this stage, the generated chromosomes are evaluated by the fitness function. Fitness function is applied to choose the optimal chromosomes for the problem. The corresponding path's frequency and node's weight values of chromosomes gene index values are utilized in the fitness function calculation. The fitness function is calculated as Eq. 2 and 3:

$$
\begin{aligned}
& \mathrm{F}^{\mathrm{v}_{\mathrm{m}} 1}=\frac{1}{\mathrm{x}} \sum_{\mathrm{x}=0}^{\mathrm{x}} \mathrm{f}_{\mathrm{x}}^{\mathrm{i}_{\mathrm{x}}} \\
& \mathrm{f}^{\mathrm{i}_{\mathrm{x}}}=\mathrm{v}\left(\mathrm{p}_{\mathrm{j}}\right)+\left(\mathrm{w}_{\mathrm{n}-1} * \mathrm{wn}\right)
\end{aligned}
$$

where, $\mathrm{F}^{\mathrm{vmI}}$ is the fitness function of the $\mathrm{I}^{\text {th }}$ chromosome generated for the vehicle $v_{m}$ and $f_{x}$ ix is the individual fitness value of the index (gene) value $I_{x}$. In E. $3, v\left(p_{j}\right)$ is the frequency value of the frequent path $p_{j}$, which is presented in the index $i_{x}$ and $W_{n-1}$ and $W n$ are the weight value of the nodes and the path $p_{j}$ is presented between 
these two nodes. The genes individual fitness value is calculated by Eq. 3 and the chromosomes fitness function is also calculated via Eq. 2. Then, the fitness is calculated for all the chromosomes and the best $\mathrm{n}_{\mathrm{c}} / 2$ chromosomes that have the highest fitness value are selected. Subsequently, the selected best chromosomes are subjected to the crossover and mutation operations.

\subsection{Crossover}

In this stage, a single point crossover is performed at a crossover rate of $R_{C}$. The gene values are exchanged between chromosomes based on the crossover rate and finally obtained $n_{c} / 2$ children chromosomes.

\subsection{Mutation}

In this stage, the children chromosomes are mutated at a mutation rate of $R_{M}$ in order to obtain $n_{c} / 2$ new chromosomes. Here, new genes are generated and substituted in the genes of previously obtained children chromosomes, where the mutation point is placed. Consequently, we obtain $\mathrm{n}_{\mathrm{c}} / 2$ new children chromosomes and $n_{c} / 2$ best parent chromosomes.

\subsection{Termination}

The above process is repeated until it reaches the utmost number of iterations I. Once it reaches I, the best $\mathrm{c}^{\mathrm{vmI}}$ chromosomes that have highest fitness value are chosen. Among the best $\mathrm{c}^{\mathrm{vml}}$ chromosomes, some chromosomes contain same index values. This replication of index values creates complexity in the further process. So, the repeated index values are taken on one occasion and the remaining repeated values are eliminated.

\subsection{Optimal Paths Training in FFBNN}

By performing the above process, now we have number of optimal paths index values for each vehicle and these optimal paths are utilized to find the vehicle future location by FFBNN. To accomplish the location prediction process, the optimal paths index values are selected from the vehicle's best chromosomes and given to the FFBNN. The Feed Forward Back Propagation Neural Network (FFBNN) is employed to perform the training and testing process. In training phase, each index value of vehicles best chromosomes and the corresponding paths nodes are given as input to the FFBNN. For example, we select one index value $i_{x}$ from the best chromosome $c^{\mathrm{vmI}}$ of the vehicle $\mathrm{v}_{\mathrm{m}}$. Here, we have taken the FFBNN input as $i_{x}$ index corresponding paths node values $n 1$ and $n 2$. In FFBNN, there is $H_{d}$ number of hidden layers and one output layer, which is an individual fitness value of the index $i_{x}$ as $f_{x}$. The
FFBNN is well trained by this optimal paths and provided an accurate fitness value for the input node values. The proposed vehicle future location prediction FFBNN structure is shown in Fig. 3.

The FFBNN contains two input units, one output units and $\mathrm{N}_{\mathrm{d}}$ hidden units. Initially, the input data is transmitted to the hidden layer and then, to the output layer. This process is called the forward pass of the back propagation algorithm. Each node in the hidden layer gets input from the input layer, which are multiplexed with suitable weights and summed. The hidden layer input value calculation function is called as bias function, which is described below Eq. 4:

$$
\mathrm{Y}_{\mathrm{i}_{\mathrm{x}}}=\beta+\sum_{\mathrm{h}=1}^{\mathrm{H}_{\mathrm{d}}}\left(\mathrm{w}_{\mathrm{h}} \mathrm{nl}_{\mathrm{i}_{\mathrm{x}} \mathrm{h}}+\mathrm{w}_{\mathrm{h}} \mathrm{n} 2_{\mathrm{i}_{\mathrm{x}} \mathrm{h}}\right)
$$

In Eq. $4, n 1_{\mathrm{i}_{\mathrm{x}}}$ and $\mathrm{n} 2_{\mathrm{i}_{\mathrm{x}}}$ are the input node values of the index $i_{x}$. The output of the hidden node is the nonlinear transformation of the resulting sum. Same process is followed in the output layer. The following Eq. 5 denotes the activation function of the output layer. The output values from the output layer are compared with target values and the learning error rate for the neural network is computed, which is given in Eq. 6:

$$
\begin{aligned}
& \alpha=\frac{1}{1+\mathrm{e}^{-Y_{\mathrm{c}^{y_{\mathrm{i}}}}}} \\
& \gamma=\frac{1}{\mathrm{H}_{\mathrm{d}}} \sum_{\mathrm{h}=0}^{\mathrm{H}_{\mathrm{d}}-1} \mathrm{D}_{\mathrm{h}}^{\mathrm{i}_{\mathrm{x}}}-\mathrm{f}_{\mathrm{h}}^{\mathrm{i}_{\mathrm{x}}}
\end{aligned}
$$

In Eq. $6, \gamma$ is the learning error rate of the FFBNN, $D_{h}{ }^{i x}$ is the desired output and $\mathrm{f}_{\mathrm{h}} \mathrm{ix}$ is the actual output. The error between the nodes is transmitted back to the hidden layer and this process is called the backward pass of the back propagation algorithm. The reduction of error by back propagation algorithm is described in the subsequent steps:

- Initially, the weights are assigned to hidden layer neurons. The input layer has a constant weight, whereas the weights for output layer neurons are chosen arbitrarily. Subsequently, the bias function and output layer activation function are computed by using the Eq. 4 and 5

- Next, the back propagation error is computed for each node and the weights are updated by using the Eq. 7:

$\mathrm{w}_{\mathrm{i}_{\mathrm{x}} \mathrm{h}}=\mathrm{w}_{\mathrm{i}_{\mathrm{x}} \mathrm{h}}+\Delta \mathrm{w}_{\mathrm{i}_{\mathrm{x}} \mathrm{h}}$ 


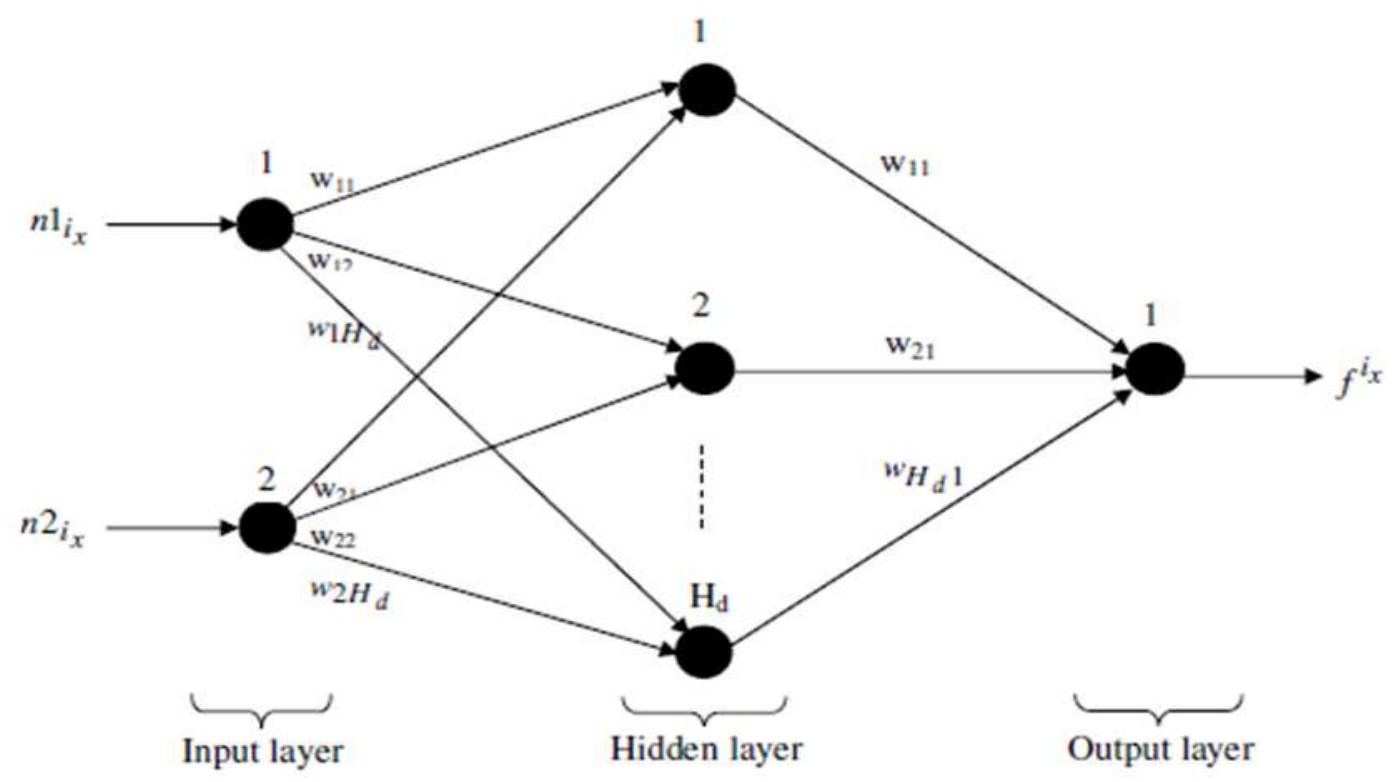

Fig. 3. Structure of optimal paths training in FFBNN

where, $\delta$ the weight $\Delta \mathrm{w}_{\mathrm{ix}} \mathrm{h}$ is changed, which is given as Eq. 8:

$$
\Delta \mathrm{w}_{\mathrm{i}_{\mathrm{x}} \mathrm{h}}=\delta . \mathrm{Y}_{\mathrm{i}_{\mathrm{h}}} \cdot \mathrm{E}^{(\varepsilon)}
$$

where, is the learning rate that normally ranges from 0.2 to 0.5 and $\mathrm{E}^{(\varepsilon)}$ is the $\mathrm{BP}$ error. The bias function, activation function and $\mathrm{BP}$ error calculation process are continued till the BP error gets reduced i.e., $\mathrm{E}^{(\varepsilon)}<0$.1. If the BP error reaches a minimum value, then the FFBNN is well trained by the paths node values for performing the vehicle location prediction. The well trained FFBNN provides an appropriate fitness values for the respective input path values.

\subsection{Vehicle Future Location Prediction by FFBNN}

The well trained FFBNN is utilized to predict the vehicles future location. Let us assume that the vehicle $\mathrm{v}_{\mathrm{m}}$ initial starting node $\mathrm{n}_{\mathrm{i}}$ is given by the user. Subsequently, all the possible paths $\mathrm{p}$ via nodes visited by the vehicle $\mathrm{v}_{\mathrm{m}}$ are given to the well trained FFBNN and we got the fitness values for all the input paths $\mathrm{p}$. Among the paths, the vehicle $\mathrm{v}_{\mathrm{m}}$ visiting next path is predicted by their fitness value output from the FFBNN, which are given as:

$\mathrm{F}_{\mathrm{v}_{\mathrm{m}}}=\max \left\{\mathrm{f}_{\mathrm{p}_{1}}, \mathrm{f}_{\mathrm{p} 2}, \cdots \mathrm{f}_{\mathrm{p}_{\mathrm{z}}}\right\}$
In Eq. 9, $f_{p z}$ is the fitness value of the path $p_{z}$ and $F_{v m}$ have the path value, which has the highest fitness value than the others. Suppose we get the path $p_{z}$ having a highest fitness value than the other paths, then this highest fitness value path is considered as a next visiting path of a vehicle $\mathrm{v}_{\mathrm{m}}$ from the current node $\mathrm{n}_{\mathrm{i}}$. In this way, the vehicles future location is predicted from the current position. The well trained FFBNN reduces the time complexity as well as gives the optimal future vehicles paths, because the training process in FFBNN is carried out by the GA algorithm. The similar procedure is followed for all vehicles to predict the future location efficiently and more accurately.

\section{RESULTS}

The proposed heuristic moving vehicle location prediction technique is implemented in the working platform of MATLAB version 7.12. The proposed heuristic technique accurately finds the moving vehicles location by finding their frequent paths. Here, all the vehicles frequent moving paths are collected and then optimal frequent paths of each vehicle are computed by the GA optimization technique. Each vehicle frequent path is trained in the FFBNN and later in performance testing, the vehicles moving location is predicted. Five vehicles frequent moving paths are noted at certain time, which is listed in the below Table 2. 


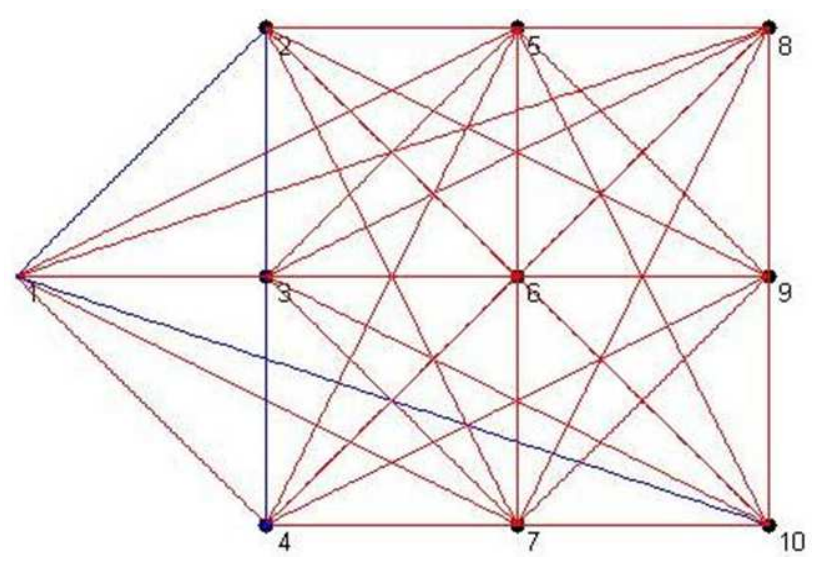

Fig. 4. Sample moving paths of vehicle A (10-4)

Table 1. Different paths obtained in three time periods

\begin{tabular}{lll}
\hline Time periods (T) & Vehicles (V) & Visited paths (p) \\
\hline t1 & v1 & $1->2->3->4$ \\
& v2 & $3->4->2->1$ \\
& v3 & $2->3->4->5$ \\
& v4 & $1->2->3->4->5$ \\
& v5 & $4->5->3>2$ \\
t2 & v1 & $2->3->4$ \\
& v2 & $3->4->2->1$ \\
& v3 & $2->3->4->5$ \\
& v4 & $1->2->3->5$ \\
t3 & v5 & $4->5->3>2$ \\
& v1 & $1->2->3->4$ \\
& v2 & $3->2->1$ \\
v3 & $2->4->5$ \\
& v4 & $2->3->4->5$ \\
& v5 & $4->3->2$ \\
\hline
\end{tabular}

Table 2. Sample frequent paths of five vehicles

\begin{tabular}{llllll}
\hline Vehicles & $\mathrm{A}$ & $\mathrm{B}$ & $\mathrm{C}$ & $\mathrm{D}$ & $\mathrm{E}$ \\
\hline Frequent paths & $1-2$ & $1-7$ & $5-9$ & $6-8$ & $10-2$ \\
& $2-5$ & $2-10$ & $7-10$ & $7-10$ & $4-10$ \\
& $3-9$ & $4-7$ & $6-10$ & $8-9$ & $2-8$ \\
\hline
\end{tabular}

Table 3. Optimal frequent paths obtained for each vehicle by proposed heuristic moving vehicle location prediction technique

\begin{tabular}{lllllllllll}
\hline Vehicles & ----A--- & $---B---$ & ---C--- & ---D--- & ---E--- \\
\hline Frequent paths & 10 & 9 & 1 & 9 & 1 & 8 & 3 & 2 & 10 & 1 \\
& 4 & 10 & 8 & 5 & 1 & 6 & 6 & 4 & 3 & 9 \\
& 4 & 2 & 2 & 3 & 4 & 6 & 6 & 4 & 4 & 6 \\
& 9 & 6 & 8 & 4 & 7 & 8 & 9 & 3 & 7 & 8 \\
& 3 & 2 & 9 & 3 & 9 & 10 & 8 & 4 & 4 & 1 \\
\hline
\end{tabular}

Table 4. Different vehicles performance accuracy results by exploiting proposed heuristic moving vehicle location prediction technique

\begin{tabular}{llllll}
\hline Vehicles & A & B & C & D & E \\
\hline Accuracy & 80 & 80 & 100 & 80 & 100 \\
\hline
\end{tabular}

Table 5. Different vehicles performance accuracy results by exploiting RBF

\begin{tabular}{llllll}
\hline Vehicles & A & B & C & D & E \\
\hline Accuracy & 80 & 80 & 40 & 40 & 20 \\
\hline
\end{tabular}

The Table 1 shows the vehicles moving paths with different starting nodes. These paths are not the most frequent path of the vehicles. So, we have to find most frequent paths for each vehicle. By using these frequent paths, the optimal frequent paths are computed and given to the FFBNN. The sample moving paths (in blue color) of vehicle A is illustrated in the following Fig. 4.

At present, we find all the vehicles optimal frequent paths and these optimal paths of each vehicle are then trained in FFBNN. This well trained FFBNN is utilized in the testing process. The proposed heuristic technique is tested with five numbers of vehicles and the predicted results are given in the following Table 3.

The proposed heuristic technique prediction accuracy is shown in the below Table 4 . The vehicle prediction accuracy is calculated by utilizing the formula.

Accuracy $=$ correctly predicted paths/total number optimal frequent paths.

The proposed vehicle path prediction performance is compared with another one classifier as RBF.

The optimal paths are given Table 3 is given to the RBF for performing the training process and the trained RBF (Yang and Zhang, 2012) is tested with moving vehicles. The prediction accuracy of RBF for five vehicles is given in Table 5.

The comparison result of both methods performance measures are given in the Fig. 5.

\section{DISCUSSION}

As can be seen from Table 4-5 and Fig. 5 our proposed technique has offered $88 \%$ mean accuracy but the RBF prediction method has given only $48 \%$ accuracy. Among the five vehicles, four vehicles prediction accuracy results are higher than the RBF method and the prediction accuracy of vehicle B is same for both methods. However, our proposed technique has not produced low accuracy result. The graphical representation of the accuracy performance results shows that our heuristic moving vehicle location prediction technique accurately finds the future moving location than the RBF method. 


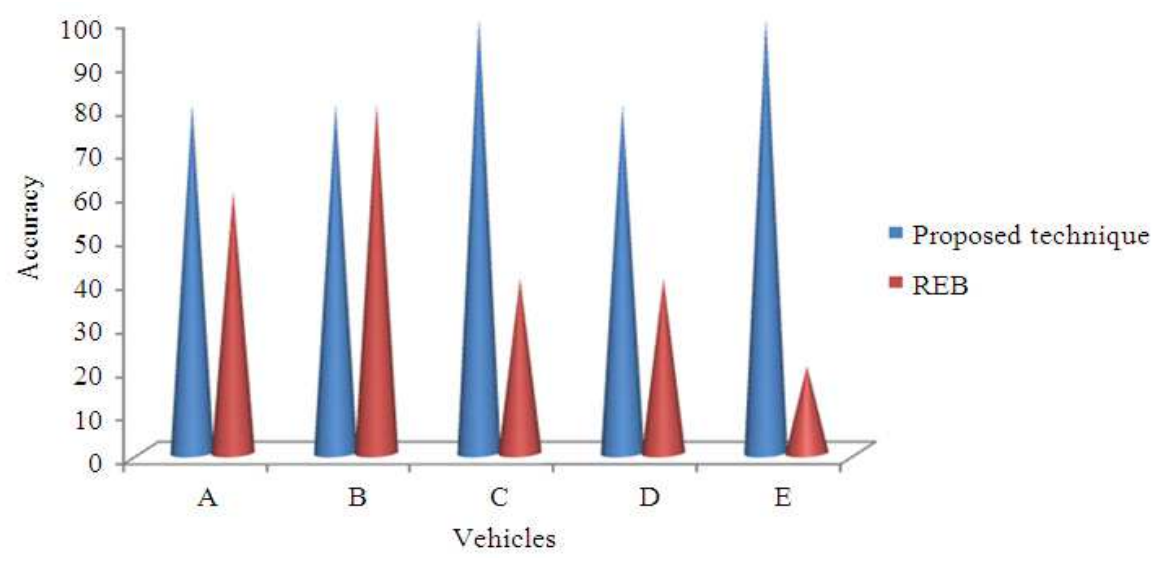

Fig. 5. Graphical representation of proposed and RBF prediction accuracy

\section{CONCLUSION}

In this study, the proposed heuristic moving vehicle location prediction algorithm was illustrated in detail with its implementation results. In the proposed methodology, the vehicles future location prediction was done using GA and FFBNN. The optimal frequent paths were computed for all vehicles through GA and the selected optimal frequent paths were utilized in the FFBNN training and testing process. All these processes have improved the performance of the proposed heuristic moving vehicle location prediction algorithm. The results have shown that the proposed heuristic algorithm has accurately found the vehicles future location with higher accuracy than the RBF classifier. Thus, our proposed heuristic algorithm has offered better performance in predicting the vehicles future location with higher accuracy. This declares that the proposed heuristic algorithm is more effective in vehicle location prediction as well as more appropriate for all the moving vehicles.

\section{REFERENCES}

1. Brakatsoulas, S., D. Pfoser, R. Salas and C. Wenk, 2005. On map-matching vehicle tracking data. Proceedings of the 31st International Conference on Very Large Data Bases, (VLDB' 05), ACM Press, pp: 853-864.

2. Brakatsoulas, S., D. Pfoser and N. Tryfona, 2004. Modeling, storing and mining moving object databases. Proceedings of the International Conference on Database Engineering and Applications Symposium, Jul. 7-9, IEEE Xplore Press, pp: 68-77. DOI: 10.1109/IDEAS.2004.1319779
3. Buang, N., A.M. Zin and M.S. Zakaria, 2011. Exploring spatial relationships for knowledge discovery in spatial data. Proceedings of the International Conference on Computer Engineering and Applications, (CEA' 11), IACSIT Press, Singapore, pp: 487-491. www.ipcsit.net/vol2/91-C137.pdf

4. Ester, M., H.P. Kriegel and J. Sander, 2001. Algorithms and applications for spatial data mining. University of Munich. http://fogo.dbs.ifi.Imu.de/Publikationen/P apers/Chapter7.revised.pdf

5. Lee, J.G., J. Han, X. Li and H. Gonzalez, 2008. TraClass: Trajectory classification using hierarchical region-based and trajectory-based clustering. Proc. VLDB Endowment, 1: 10811094.

http://dl.acm.org/citation.cfm?id=1453972

6. Li, D. and S. Wang, 2005. Concepts, Principles and Applications of Spatial Data Mining and Knowledge Discovery. Proceedings of the International Symposium on Spatio-Temporal Modeling, Aug. 27-29, Beijing, China, pp: 1-13. http://www.isprs.org/proceedings/XXXVI/ 2W25/source/CONCEPTS PRINCIPLES AND APPLICATIONS_OF_SPATTIAL_DATA_MINI NG_AND_KNOWLEDGE_DISCŌVERY.pdf

7. Li, X., J. Han and S. Kim, 2006. Motion-alert: Automatic anomaly detection in massive moving objects. Intell. Secu. Inform., 3975: 166-177. DOI: 10.1007/11760146_15 
8. Liu, H. and M. Schneider, 2010. Detecting the topological development in a complex moving region. J. Mul. Process. Technol., 5: 1-25.

9. Liu, H. and M. Schneider, 2011. Tracking continuous topological changes of complex moving regions. Proceedings of the ACM Symposium on Applied Computing, Mar. 2124, ACM Press, New York, pp: 833-838. DOI: $10.1145 / 1982185.1982366$

10. Mennis, J. and D. Guo, 2009. Spatial data mining and geographic knowledge discoveryAn introduction. Comput. Environ. Urban Syst., 33: 403-408.

DOI: 10.1016/j.compenvurbsys.2009.11.001

11. Monreale, A., F. Pinelli, R. Trasarti and F. Giannotti, 2009. WhereNext: A location predictor on trajectory pattern mining. Proceedings of the 15th ACM SIGKDD International Conference on Knowledge Discovery and Data Mining, Jun. 28-Jul. 01, ACM Press, New York, USA., pp: 637-646. DOI: $10.1145 / 1557019.1557091$

12. Pena, J.H. and M.Y. Santos, 2011. Representing, storing and mining moving objects data. Proceedings of the World Congress on Engineering, Jul. 6-8, IEEE Xplore Press, London, U.K., pp: 1-6. http://www.iaeng.org/publication/WCE2011/W CE2011_pp1823-1828.pdf

13. Roddick, J.F., E. Hoel, M.J. Egenhofer, D. Papadias and B. Salzberg, 2004. Spatial, temporal and spatio-temporal databases-Hot issues and directions for $\mathrm{PhD}$ research. SIGMOD Rec., 33: 126-131. DOI: 10.1145/1024694.1024724

14. Satpathy, S., L. Sharma, A.K. Akasapu and N. Sharma, 2011. Towards mining approaches for trajectory data. Int. J. Adv. Sci. Technol., 2: 3843.

http://www.svpublishers.co.uk/download/i/mark dl/u/4008228453/4550179407/Paper-5.pdf

15. Shaw, A.A. and N.P. Gopalan, 2011. Frequent pattern mining of trajectory coordinates using apriori algorithm. Int. J. Comput. Appl., 22: 1-7. http://www.ijcaonline.org/volume22/number9/p xc3873094.pdf

16. Yang, L. and J. Zhang, 2012. Prediction study on anti-slide control of railway vehicle based on RBF neural networks. Phys. Proc., 25: 911-916. DOI: 10.1016/j.phpro.2012.03.177 